\title{
BMJ Global Health Hierarchy of qualities in global health partnerships: a path towards equity and sustainability
}

\author{
Simone H Schriger (1) , ${ }^{1}$ Agnes Binagwaho, ${ }^{2}$ Moses Keetile, ${ }^{3}$ Vanessa Kerry, ${ }^{4,5}$ \\ Joel M Mubiligi, ${ }^{6}$ Doreen Ramogola-Masire, ${ }^{7,8}$ Michelle Roland, ${ }^{9}$ Frances K Barg, ${ }^{8}$ \\ Corrado Cancedda (i) ${ }^{8}$
}

\begin{abstract}
To cite: Schriger SH, Binagwaho A, Keetile M, et al. Hierarchy of qualities in global health partnerships: a path towards equity and sustainability. BMJ Global Health 2021;6:e007132. doi:10.1136/ bmjgh-2021-007132
\end{abstract}

Handling editor Seye Abimbola

Received 12 August 2021 Accepted 3 December 2021

\section{Check for updates}

\section{Author(s) (or their} employer(s)) 2021. Re-use permitted under CC BY-NC. No commercial re-use. See rights and permissions. Published by BMJ.

For numbered affiliations see end of article.

Correspondence to Dr Corrado Cancedda; ccancedd@gmail.com

\section{ABSTRACT}

Despite the exponential growth of global health partnerships (GHPs) over the past 20 years, evidence for their effectiveness remains limited. Furthermore, many partnerships are dysfunctional as a result of inequitable partnership benefits, low trust and accountability and poor evaluation and quality improvement practices. In this article, we describe a theoretical model for partnerships developed by seven global health experts. Through semistructured interviews and an open-coding approach to data analysis, we identify 12 GHP pillars spanning across three interconnected partnership levels and inspired by Maslow's hierarchy of needs. The transactional pillars are governance, resources and expertise, power management, transparency and accountability, data and evidence and respect and curiosity. The collaborative pillars (which build on the transactional pillars) are shared vision, relationship building, deep understanding and trust. The transformational pillars (which build on the collaborative pillars and allow partnerships to achieve their full potential) are equity and sustainability. The theoretical model described in this article is complemented by reallife examples, which outline both the cost incurred when GHPs fail to live up to these pillars and the benefits gained when GHPs uphold them. We also provide lessons learnt and best practices that GHPs should adopt to further increase their strength and improve their effectiveness in the future. To continue improving health outcomes and reducing health inequities globally, we need GHPs that are transformational, not just rhetorically but de facto. These actualised partnerships should serve as a catalyst for the greater societal good and not simply as a platform to accrue and exchange organisational benefits.

\section{BACKGROUND}

Global health partnerships (GHPs) have grown exponentially over the past 20 years. ${ }^{12}$ We define a GHP as a multiyear working relationship between two or more organisations from different countries (eg, governments, multilateral organisations, academic institutions, non-governmental organisations (NGOs), donors and local community and grassroots organisations). This exponential
Summary box

- Empirical evidence for the effectiveness of globa health partnership remains limited despite the exponential increase in partnerships since the early 2000s.

- This article describes a theoretical model for partnerships, which is inspired by Maslow's hierarchy of needs and comprised of 12 pillars spanning across three interconnected partnership levels (transactional, collaborative and transformational).

- Each level in the model builds on the previous; the transactional level (including governance, resources and expertise, power management, transparency and accountability, data and evidence and respect and curiosity) gives way to the collaborative level (shared vision, relationship building, deep understanding and trust), which leads to the transformational level (equity and sustainability)

- This model is complemented by real-life examples, detailing the cost of not living up to these pillars, the benefits of upholding them, and best practices to support partnerships reaching the transformational level.

growth has occurred due to increased availability of funds through new donors (such as the US President's Emergency Plan for AIDS Relief, among others) and as a response to the devastating impact of HIV/AIDS in SubSaharan Africa.

GHPs are important for two reasons: (a) many countries do not yet have all the resources necessary to meet the public health needs of their populations, (b) some challenges (such as the 2015 Ebola epidemic in West Africa and the current COVID-19 pandemic) span multiple nations such that no country can tackle them in isolation. The critical importance of partnerships in international development is illustrated by their repeated mention in the Sustainable Development Goals (SDGs). ${ }^{3-6}$ 
Unfortunately, empirical evidence for the effectiveness of GHPs is limited. ${ }^{1278}$ Anecdotally, many partnerships fail to achieve their goals because of inequitable partnership benefits, low trust and accountability and poor evaluation and quality improvement practices. Further evidence is needed to determine whether overcoming these dysfunctions will aid in advancing health globally. ${ }^{910}$

\section{DEVELOPING A THEORETICAL MODEL FOR GHPS}

We carried out semistructured interviews with seven global health experts, who were identified using a purposive sampling technique with inclusion criteria, including having at least 10 years of experience and having managed at least one multimillion dollar programme or initiative. All identified experts agreed to participate and were from a mix of high-income, middle-income and lowincome countries. Additionally, experts were all either currently working or had previously worked at a government agency or ministry of health, donor organisation, academic institution or NGO.

Semistructured interviews were led by experts in qualitative research and centred around three questions: 'What are the key qualities and ideals of a global health partnership?', 'What are the benefits of upholding these qualities and ideals in terms of partnership effectiveness and other outcomes?' and 'What are the costs of failing to live up to these qualities and ideals?' All experts were interviewed two times. Initial interviews focused on the above questions and were coded using an open coding approach to identify recurring themes within the transcripts and develop a codebook. Follow-up interviews were used to validate preliminary themes and generate additional ideas. Each interview lasted $45-60 \mathrm{~min}$ and was conducted over a videoconferencing platform. Interviews were audio-recorded, transcribed and coded in NVIVO V.12.0 using a thematic analysis approach. ${ }^{11}$ After the follow-up interviews, theoretical saturation was reached and, thus, no further interviews were conducted. Themes and subthemes were identified and ultimately a theoretical model for successful GHPs was developed based on the 14 interviews. Finally, after writing the first draft of the manuscript and presenting our model, all experts were invited to provide specific examples of lessons learnt and best practices that facilitate GHP success.

\section{TWELVE PILLARS FOR SUCCESSFUL GHPS}

From the interviews, we identified 12 themes, which we conceptualised as pillars. Each pillar represents an essential quality of a successful GHP: (a) data and evidence, (b) deep understanding, (c) equity, (d) governance, (e) power management, (f) relationship building, (g) resources and expertise, (h) respect and curiosity, (i) shared vision, (j) sustainability, (k) transparency and accountability and (l) trust (table 1). During the initial interview, all experts also provided real-life examples about the costs of failing to live up to these pillars and the benefits of upholding them.
The real-life examples and the interconnected nature of the 12 pillars led to the development of a theoretical model for GHPs, inspired by Maslow's hierarchy of needs (figure 1). Some pillars (governance, resources and expertise, power management, transparency and accountability, data and evidence and respect and curiosity) must be present in the earliest stages of a partnership (transactional level). Other pillars (shared vision, relationship building, deep understanding and trust) develop over time and allow the partnership to mature from the transactional level to a collaborative level. The last two pillars (equity and sustainability) allow partnerships to mature to the transformational level (analogous to Maslow's self-actualisation stage) and achieve their highest potential. In the model, the pillars occur sequentially, with transactional pillars first leading to collaborative pillars, and collaborative pillars leading later to transformational pillars. Conversely, non-upholding of some pillars at an earlier stage prevents the upholding of other pillars at the following stage, leading to partnership underperformance. The model emphasises how all ingredients need to be in place for partnerships to achieve their greatest potential and how establishing a strong and effective partnership is a time-consuming and resource-intensive endeavour.

When a partnership is transactional, the primary focus is on organisational benefits and the partner with the most power usually accrues the most benefits. When a partnership is collaborative, the focus is still not only on organisational benefits but also on the greater societal good (in the case of global health, the continued improvements in health outcomes and reductions in health inequities) while the benefits are distributed more fairly among the partners. Finally, when a partnership is transformational, the focus is primarily on the greater societal good, which requires significant redistribution of power among partners and organisations to change in more fundamental ways than just by tweaking or revising their policies and practices.

\section{THE COST OF PARTNERSHIPS NOT LIVING UP TO THE 12 PILLARS}

All seven global health experts gave real-life examples of partnerships, where a failure to live up to one or more of the pillars had a detrimental effect on the partnership's ability to reach the transformational level.

\section{"I never saw an effective development partner coordination process... Harder to get things done"}

Governance and accountability challenges within a partnership lead to a lack of shared vision, which can lead to sustainability challenges

- When international partners do not coordinate among themselves, they often overwhelm national governments trying to hold them accountable. Simultaneously, the willingness and capacity of some national governments to play this critical coordinating 
Table 1 Levels of GHPs and associated pillars

Transactional level

Governance Establishment of policies and processes for planning, decision-making and programme implementation with equitable representation and selection of leaders and implementers

Resources and Adequate amount, quality and diversity of resources and expertise cumulatively brought by all organisations expertise to the partnership

Transparency and Commitment to shared goals, reliability, willingness to change, honesty about one's concerns, ideas and accountability needs

Power management Acknowledgement of mismatches in levels of agency and vulnerability among partners and pursuit of some practices to redistribute resources, expertise and power

Data and evidence Ongoing evaluation of partnership quality and effectiveness and adoption of corrective measures when necessary

Respect and curiosity Non-judgmental, open-minded, and thoughtful assessment of each partner's history, culture, power, strengths and limitations

\begin{tabular}{|c|c|}
\hline \multicolumn{2}{|l|}{ Collaborative level } \\
\hline Shared vision & $\begin{array}{l}\text { Common understanding of a partnership's desired outcomes and ultimate goals, each partner's } \\
\text { responsibility, and each partner's expected benefits }\end{array}$ \\
\hline Relationship building & Ongoing strengthening of organisational ties and improvement of the ways in which partners work together \\
\hline Deep understanding & $\begin{array}{l}\text { Profound appreciation for each partner's values, priorities, level of agency (or control over partnership } \\
\text { outcomes and goals) and vulnerability (or consequences in case of partnership failure or under- } \\
\text { performance) }\end{array}$ \\
\hline Trust & $\begin{array}{l}\text { Firm belief in other partner's good intentions, commitment, reliability, ability, honesty, and integrity and solid } \\
\text { track record of behaviour by all partners that justifies this belief }\end{array}$ \\
\hline \multicolumn{2}{|l|}{ Transformational level } \\
\hline Equity & $\begin{array}{l}\text { Intense prioritisation of needs of partners (including patients, households, and communities) with } \\
\text { greatest vulnerability and lowest level of agency, resources, expertise, and significant reduction in power } \\
\text { mismatches }\end{array}$ \\
\hline Sustainability & $\begin{array}{l}\text { Maintenance of partnership outcomes and goals beyond the duration of the partnership as a result of } \\
\text { organisational capacity strengthening and community empowerment }\end{array}$ \\
\hline
\end{tabular}

GHPs, global health partnerships.

role varies and does not necessarily relate to a country's resource level.

- The timeline of one partner is often not be compatible with that of other partner(s). For example, national governments function on a year-by-year basis because of their budgeting process and face intense pressure from their parliaments to show quick results for not only health, but other sectors as well. It is very difficult for them to commit to long-term strategic plans and devote additional resources to health. At the same time, international partners face uncertainty as well, are scrutinised and held accountable by governance

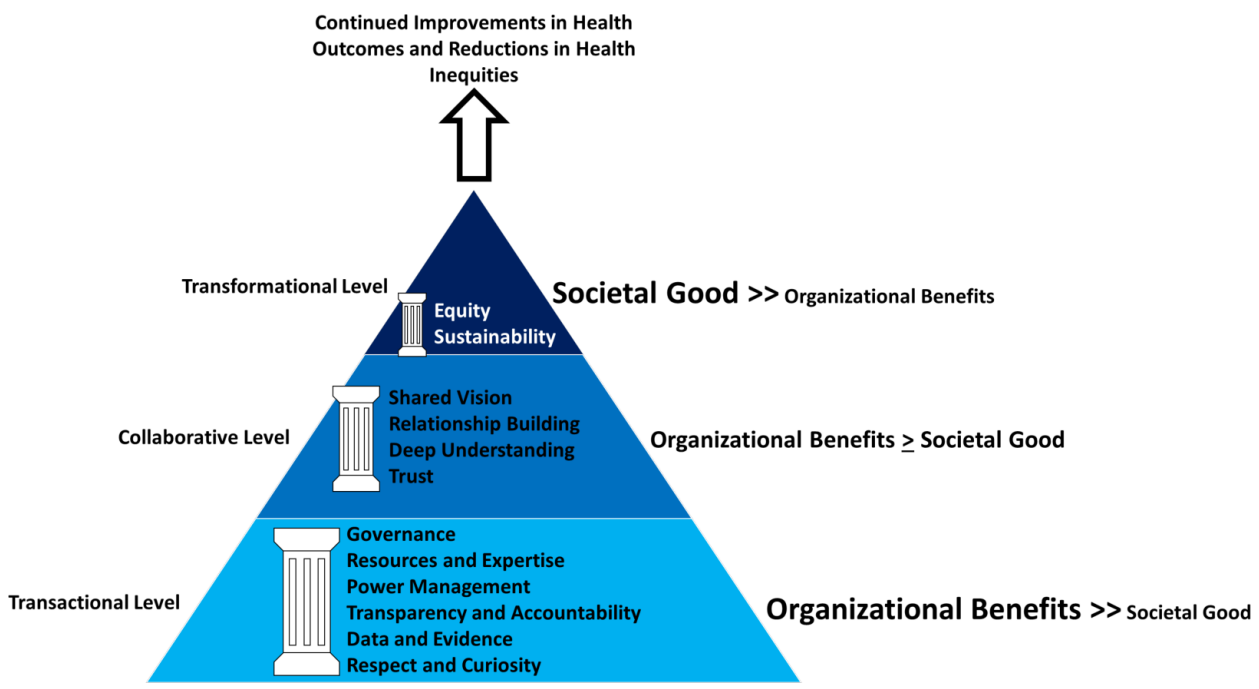

Figure 1 Theoretical model for global health partnerships. 
bodies and cannot guarantee that they will sustain their contributions beyond just a few years.

"Because of the turnover gap and human resources gaps... Support (of) the health center is not happening..." Governance challenges can lead to excessive staff turnover and poor performance, which can lead to sustainability challenges

- When health professionals in the public sector leave in large numbers or too frequently, both the availability and quality of health service delivery deteriorate. Furthermore, partner organisations providing training, mentorship and supervision to these health professionals fail to have a long-term and sustained impact. Conversely, excessive turnover of personnel within a partner organisation can lead to insufficient and inconsistent training and oversight of health professionals in the public sector.

\section{"Sponsor(s) sometimes they have their own hidden agenda" Power management and accountability challenges can lead to lack of shared vision and trust, which can lead to sustainability challenges}

- International donors and NGOs often want to establish global systems for data collection and analysis because they want to make meaningful comparisons across countries and are under pressure to show results quickly. As a result, these partners often actively hinder or fail to leverage and capacitate existing local systems and instead collect and analyse data that is more relevant to them than to local partners.

- It is critical for national governments to be in charge. In many countries, unfortunately, national governments have to alter their strategy and reluctantly accept the demands of international partners with their own agenda.

\section{'We have had a couple partners where the politics have been really fraught'}

Power management challenges can also lead to accountability challenges, which can lead to equity challenges

- Some partners have greater freedom to disengage if their priorities change, and less to lose when a project fails or challenges arise. For example, during the Ebola epidemic in West Africa, one of the donors had specifically asked an international NGO to work in an Ebola treatment unit operated by the Ministry of Health, even though at the time, there were limited infection control processes in place. The NGO leadership thought that they could help make the facilities safer, and believed it was the right thing to do. However, when one of the NGO's international staff members became sick, the donor threatened to withdraw their funding. The donor did not have the same reaction when, earlier on, two staff members from the Ministry of Health became sick. The donor's behaviour led to a deep sense of betrayal.

- Often, partners are subject to the changing whims and politics of other partners. In one project, two other partners decided to split and stop coordinating. This divide occurred along political lines and because each partner had decided to prioritise their self-interest over that of the project. Suddenly, there were not enough resources to achieve the project's goals. One of the organisations participating in the partnership was caught in the middle of the dispute and unfairly blamed for the project's failures. Additionally, this organisation had to significantly alter its strategy, get out of two countries where it had previous commitments, and fire some of its staff.

\section{'And sometimes because it's not our culture... it's going to rub someone the wrong way'}

Lack of respect and deep understanding can lead to trust challenges, which can lead to both equity and sustainability challenges

- Many international partners act as if they know better than local partners and have all the solutions. This assumption of superiority can be quite overt but can also be more implicit or very subtly stated. Even wellmeaning international partners are often unaware of these dynamics or face-intense pressure to perform quickly and at scale, which can overwhelm the need for respect and deep understanding. Local partners find it deeply offensive but might not feel empowered to speak out. As a result, projects of limited or no value are often implemented.

-Whenever partners fail to immediately address disruptions in communication, resentment builds and project implementation slows down. Unfortunately, partners are often unwilling to have uncomfortable conversations. Unacknowledged power differences among the partners limit the chance of maintaining open and honest communication. It does not help when international partners believe that local partners should feel indebted to them.

- Understanding and respecting local cultures are of critical importance. International partners often have an individualistic rather than a collectivistic approach. Certain behaviours, such as being confrontational and disrespecting a colleague in public, are unacceptable to local partners and have doomed many projects.

\section{THE BENEFITS OF PARTNERSHIPS UPHOLDING THE 12 PILLARS}

All seven global health expert practitioners also gave real-life examples, where partnerships that managed to uphold one or more of the pillars were able to reach the transformational level and achieve their goals.

\section{'If you want to help a group of humans, (you) need to cultivate their ownership...'}

Strong governance and agreeing on a shared vision can lead to sustainability of both partnership activities and outcomes

- The government of one of the expert's home country displayed high levels of ownership, was able to articulate clearly what their needs were and was able to lead and coordinate the partners and leverage synergies among 
them. However, sustainability cannot be achieved in one generation and requires the willingness by some of the partners to work themselves out of a job.

\section{'It wasn't perfect by any stretch of the imagination but was the right thing to do'}

The collaborative pillars (trust, deep understanding, relationship building and accountability) are critically important to achieving equity through systemic and organisational change

- A high-impact training programme was implemented by working through existing systems and organisations, and not by establishing new ones (as is often the norm in global health). This was possible because all partners took the time to build a relationship, understand and trust each other and commit to a shared vision. Even though many of the existing systems and organisations were not initially set up to implement the programme, the partners were able to meet the challenge and pursue the necessary organisational and systemic changes.

\section{'We tried to stay very flexible and met them in that expectation'}

Taking the time to understand one partner's needs, being transparent about challenges and being accountable to addressing them is critically important for sustainability

- In one project, local academic institutions ended up needing much greater support than originally planned in order to receive accreditation. When their partners agreed to provide such support and meet their expectations, the academic institutions became fully committed to the project and performed beautifully over the following years.

\section{'The country can continue with the kind of work that was initially set by the partnership' \\ Strong governance and judicious use of the partnership's \\ resources and expertise can also lead to a shared vision, which leads to sustainability}

- The government of one expert's home country was able to establish strong partnerships with multiple foreign governments and international organisations across a variety of projects, from short-term medical missions to long-term research endeavours. Regardless of their focus, all projects were set up, such that when the seed funding from the partnership ended, the government was able to pick up the cost of sustaining each project's activities and outcomes. Sustainability was not not only financial but also operational as the management of staff, procedures, equipment, supplies and infrastructure was gradually taken over by the government.

\section{'True empowerment of local staff..., all of those pieces lead to success'}

Strong governance and enlightened management of power dynamics within the partnership can have transformational effects

- In one of the countries where one of the experts worked, a new and complex set of normative guidelines from an international standard-setting organisation was pushed forward by establishing a core team of local staff, pairing this team with a supporting team of international staff and establishing working groups (tasked with studying, adapting and promoting the adoption of these guidelines) across concentric levels of engagement (from the core team to government officials and local communities).

\section{'So there is a moving target that is coming, but moving forward to where the government is ready'}

Respect and curiosity, transparency and accountability and judicious use of the partnership's resources and expertise can lead to health equity

- The Ministry of Health of one of the expert's country of origin was able to resist the pressure by many international partners to over burden the health system (and especially the existing cadre of community health workers) with too many responsibilities at once and opted instead to strengthen the capacity of the health system gradually. This was done by focusing first on the delivery of maternal and child health services, and later by expanding health service delivery to include mental health and other noncommunicable diseases.

\section{LESSONS LEARNED FROM AND BEST PRACTICES TO BE ADOPTED BY SUCCESSFUL GHPS}

The seven global health experts identified several best practices that had either been critical to the success of previous GHPs or that ought to be adopted by partnerships going forward (table 2). The main theme recurring across all interviews was that all partners have a responsibility to consistently question and re-evaluate how they function and to improve their performance when necessary, regardless of their role in the partnership or how much power they hold. Both national and international partners must be willing to significantly alter their practices to increase the chances that a partnership will achieve its goals. Additionally, all partners should proactively monitor the quality and effectiveness of their partnerships and take corrective measures when necessary.

Governments need to develop and implement longterm national strategic plans for health effectively and efficiently, health facilities need to provide high-quality and compassionate care, and communities should be active participants in tackling diseases and their risk factors. When they fail to do so, they should be held accountable.

As importantly (and less frequently stated), other types of partners should equally be held accountable and improve their performance. For example, donors need to be more transparent, reliable and open to giving money away differently (for longer periods of time, with fewer strings attached and in a way that is aligned with a country's or region's burden of disease), while the WHO needs to expand its strategic and technical advisory role. 
Table 2 Lessons Learnt from and best practices to be adopted by successful GHPs

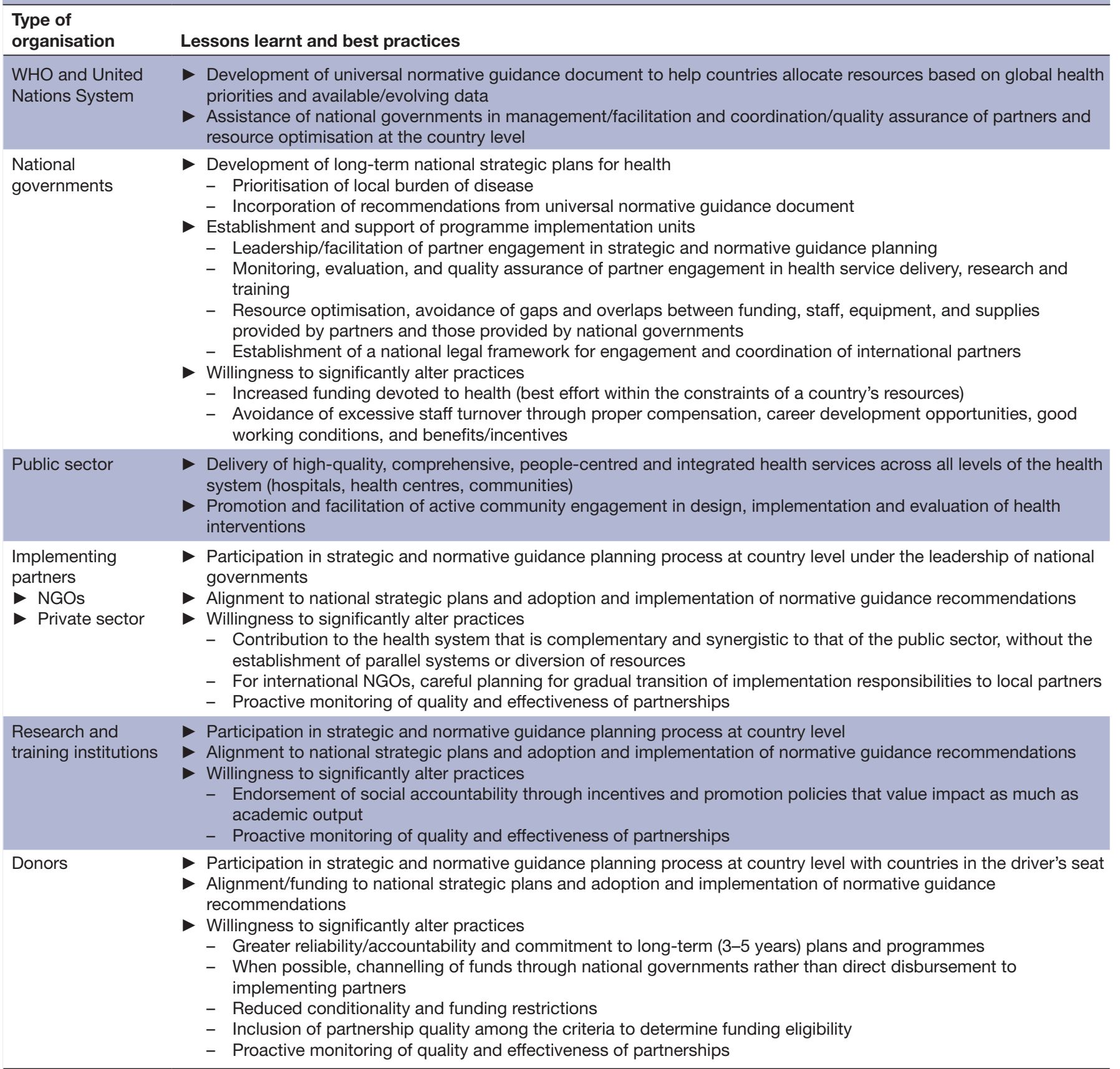

GHPs, global health partnerships; NGOs, non-governmental organisations.

Furthermore, academic institutions need to reward service and impact as much as they reward individual or organisational success, while the private sector and NGOs need to work closely with ministries of health to avoid the creation of parallel systems for health service delivery. Finally, international NGOs need to carefully plan and gradually transfer their responsibilities to the public sector or local implementing partners until their presence is no longer required (or they transition to a new set of tasks).

\section{CONCLUSION}

The scale and depth of the existing global health challenges require strong and effective partnerships across different organisations and countries. SDG17 emphasises the critical role that partnerships must play in achieving the 13 health targets within SDG3 and multiple targets within other SDGs. ${ }^{6}$ To be successful, partnerships require proactive cultivation and nurturing, and organisations should put as much effort into them as they put into the advancement of their bottom-line.

The Partnering Initiative, the Tropical Health and Education Trust and the European Esther Alliance (among others) have all released reports and studies that make a compelling and evidence-based case for partnerships. ${ }^{12-14}$ However, even though consensus within the global health community is growing, it is unclear whether 
even organisations that are in theory, the most supportive have changed their practices enough to improve the quality and effectiveness of their partnerships.

This study sought to add to the existing body of knowledge on GHPs. Seven global health expert practitioners identified 12 pillars for successful GHPs and provided real-life examples about the cost of failing to uphold them and provided examples of lessons learnt from and best practices to be adopted by successful GHPs.

What became apparent from the interviews is that many (but not all) partnerships remain at the transactional or (at best) collaborative level, even though the rhetoric surrounding GHPs is often transformational in nature. Most importantly, to achieve the SDG targets or tackle the ongoing COVID-19 epidemic (or future pandemics), the global health community needs partnerships that are transformational de facto and not just rhetorically.

What also became apparent is that there is a real cost in using partnerships primarily to advance one's organisational bottom-line (especially when the bottom line is that of international partners) or to trade benefits among organisations. This cost might explain some of global health's persistent challenges. The real-life examples provided by the expert practitioners serve as a testimony of the many ways in which suboptimal and dysfunctional partnerships hurt patients and communities around the world and waste valuable and scarce resources and expertise.

How then can the quality of GHPs be improved? The interviews point to the fact that all (not just some) partners may have to change how they operate or are incentivised in ways that are necessary and at times may be uncomfortable or even threatening. Traditionally, the burden of changing (captured by the term 'capacity building') has fallen on the partners with the least amount of power (underfunded ministries of health, understaffed health facilities, poor and under-resourced communities).$^{15}$ In a transformational partnership, the burden of changing and of building one's capacity is democratised and spread equitably across all partners.

This study has limitations. Although they came from diverse backgrounds, we incorporated the perspectives of seven experts who came from only three countries, which may not comprehensively represent the perspective of all global health experts. It is possible that interviewing a larger number of participants from a larger number of countries would have expanded our findings to include additional pillars, though in our sample, theoretical saturation was reached. Despite these limitations, the findings from our interviews reiterate the critical importance of partnerships in global health. Furthermore, our theoretical model makes the case for establishing partnerships, where (a) partners with more power (including donors) are willing to give up control and incur some risks, (b) partners with less power acquire agency and are able to play a bigger role in advancing the partnership's goals and (c) all partners do not take advantage of other partners' vulnerabilities but act instead with compassion, accountability, and trustworthiness.

Sadly, not nearly enough GHPs live up to these qualities. The time has come for GHPs to finally achieve their greatest potential and serve as a catalyst for the deep systemic change within the global health ecosystem that will lead to sustained improvements in health outcomes and reductions in health inequities for all people.

\section{Author affiliations}

${ }^{1}$ Department of Psychology, School of Arts and Sciences, University of

Pennsylvania, Philadelphia, Pennsylvania, USA

2University of Global Health Equity, Kigali, Rwanda

${ }^{3}$ Ministry of Health \& Wellness, Gabarone, Botswana

${ }^{4}$ Center for Global Health, Massachusetts General Hospital, Boston, Massachusetts, USA

${ }^{5}$ Department of Global Health and Social Medicine, Harvard Medical School, Boston, Massachusetts, USA

${ }^{6}$ Partners In Health/Inshuti Mu Buzima, Kigali, Rwanda

${ }^{7}$ University of Botswana, Gaborone, Botswana

${ }^{8}$ For Frances Barg: Department of Family Medicine and Community Health; For Corrado Cancedda and Doreen Ramogola Masire: Center for Global Health; For Corrado Cancedda only: Division of Infectious Diseases/Department of Medicine, Perelman School of Medicine, University of Pennsylvania, Philadelphia, Pennsylvania, USA

${ }^{9}$ Sutter Medical Group, An Affiliate of Sutter Medical Foundation, Jackson, California, USA

Contributors SS: conceptualisation, data curation, formal analysis, methodology, project administration, writing — original draft, writing — review and editing (accessed/verified data). AB, MK, VK, DR-M, MR: conceptualisation, writing—review and editing. FKB: conceptualisation, data curation, formal analysis, methodology, supervision (accessed/verified data). CC: conceptualisation, funding acquisition, investigation, methodology, resources, supervision, writing —original draft, writing - review and editing. CC will serve as guarantor

Funding The authors have not declared a specific grant for this research from any funding agency in the public, commercial or not-for-profit sectors.

\section{Competing interests None declared.}

\section{Patient consent for publication Not applicable.}

Ethics approval This study involves human participants but We obtained ethics approval through the University of Pennsylvania IRB (IRB protocol 833899). Participants provided informed consent before participating in interviews. exempted this study Participants gave informed consent to participate in the study before taking part.

Provenance and peer review Not commissioned; externally peer reviewed. Data availability statement Data are available upon request.

Open access This is an open access article distributed in accordance with the Creative Commons Attribution Non Commercial (CC BY-NC 4.0) license, which permits others to distribute, remix, adapt, build upon this work non-commercially, and license their derivative works on different terms, provided the original work is properly cited, appropriate credit is given, any changes made indicated, and the use is non-commercial. See: http://creativecommons.org/licenses/by-nc/4.0/.

\section{ORCID iDs}

Simone H Schriger http://orcid.org/0000-0002-0662-5867

Corrado Cancedda http://orcid.org/0000-0002-0214-955X

\section{REFERENCES}

1 Jones A. Envisioning a global health partnership movement. Global Health 2016;12:1.

2 Ritman D. Health partnership research and the assessment of effectiveness. Global Health 2016;12:43.

3 Bekker L-G, Alleyne G, Baral S, et al. Advancing global health and strengthening the HIV response in the era of the sustainable development goals: the International AIDS Society-Lancet Commission. Lancet 2018;392:312-58. 
4 Wang $\mathrm{H}$, Abbas KM, Abbasifard M, et al. Global age-sexspecific fertility, mortality, healthy life expectancy (HALE), and population estimates in 204 countries and territories, 1950-2019: a comprehensive demographic analysis for the global burden of disease study 2019. Lancet 2020;396:1160-203.

5 Martinez R, Lloyd-Sherlock P, Soliz P, et al. Trends in premature avertable mortality from non-communicable diseases for 195 countries and territories, 1990-2017: a population-based study. Lancet Glob Health 2020;8:e511-23.

6 The Sustainable Development Goals [Internet]. Available: https://sust ainabledevelopment.un.org [Accessed July 2021].

7 Edwards S, Ritman D, Burn E. Towards a simple typology of international health partnerships. Global Health 2015;11:1-7.

8 Kelly E, Doyle V, Weakliam D. A rapid evidence review on the effectiveness of institutional health partnerships. Global Health 2015;11:1-0
9 Abimbola S, Pai M. Will global health survive its decolonisation? Lancet 2020;396:1627-8.

10 Ruckert A, Labonté R. Public-private partnerships (ppp s) in global health: the good, the bad and the ugly. Third World $Q$ 2014;35:1598-614

11 Gareth T, Hayfield N, Clarke V. Thematic analysis. In: The SAGE Handbook of qualitative research in psychology. 2nd ed. SAGE, 2017: 17-37.

12 The Partnering Initiative [Internet]. Available: https://thepartneringini tiative.org/ [Accessed July 2021].

13 The Tropical Health and Education Trust [Internet]. Available: https:// www.thet.org/ [Accessed July 2021].

14 The European Esther Alliance [Internet]. Available: https://esther.eu/ [Accessed July 2021].

15 Ward CL, Shaw D, Sprumont D, et al. Good collaborative practice: reforming capacity building governance of international health research partnerships. Global Health 2018;14:1-6. 